\title{
Triagem para hepatite $C$ em unidades de urgência e emergência: revisão sistemática
}

\author{
Screening for hepatitis $C$ in urgent and emergency units: a systematic review \\ Tamizaje de la hepatitis $C$ en unidades de urgencia y emergencia: revisión sistemática
}

Como citar este artigo:

Gardona RGB, Barbosa DA, Ferraz MLG. Screening for hepatitis C in urgent and emergency units: a systematic review. Rev Esc Enferm USP. 2020;54:e03611. doi https://doi.org/10.1590/S1980-220X20180450-03611

\section{Rodrigo Galvão Bueno Gardona ${ }^{1}$}

Dulce Aparecida Barbosa²

Maria Lucia Gomes Ferraz ${ }^{1}$

${ }^{1}$ Universidade Federal de São Paulo, Escola Paulista de Medicina, São Paulo, SP, Brasil.

${ }^{2}$ Universidade Federal de São Paulo, Escola Paulista de Enfermagem, São Paulo, SP, Brasil.

\begin{abstract}
Objective: Identifying studies that performed screening for hepatitis $\mathrm{C}$ and that also assessed the virus prevalence in urgency and emergency unit users. Method: A systematic review performed on the LILACS, MEDLINE databases via PubMed, SciELO and Virtual Health Library. Results: After careful search and analysis, 19 publications were part of the results, in compliance with the pre-defined criteria. Publication dates were concentrated between 1992 and 2018. The main journal to publish such articles was the Annals of Emergency Medicine (26.31\%). Regarding the origin of publications, the following stand out: United States (63.15\%), Europe (31.57\%), and Asia (5.28\%). Regarding prevalence, in the United States, a variation between $1.4 \%$ to $18 \%$ was observed. In Europe, in turn, the prevalence ranged from 0 to 5\%. In the Asian study, the prevalence was $1.8 \%$. Conclusion: The screening performed in urgent and emergency units proved to be efficient in identifying new cases, especially when associated with the age factor, which proves to be superior to the strategy based only on risk factors. Thus, for greater effectiveness, the association of three strategies is recommended: screening location, age group, and risk factors.
\end{abstract}

DESCRIPTORS

Hepatite C; Mass Screening; Ambulatory Care; Prevalence; Systematic Review. 


\section{INTRODUÇÃO}

Até recentemente, estimava-se que 170 milhões de pessoas no mundo estivessem infectadas com o vírus da hepatite $\mathrm{C}(\mathrm{HCV})^{(1)}$. Atualmente, esta estimativa é de 71 milhões de pessoas com infecção pelo HCV na população global( ${ }^{(2)}$.

Um inquérito que avaliou a prevalência da hepatite $\mathrm{C}$ nas capitais brasileiras identificou uma taxa de $1,38 \%$ na faixa etária de 10 a 69 anos $^{(3)}$. A prevalência atual, entretanto, estima-se ser de $0,7 \%{ }^{(4)}$.

O Ministério da Saúde lançou recentemente um plano para eliminar a hepatite $\mathrm{C}$ até 2030 , com base nas recomendações da Organização Mundial da Saúde (OMS) ${ }^{(5-6)}$.

Essa campanha visa identificar pacientes infectados pelo vírus e, com o tratamento, prevenir as principais complicações relacionada à doença, tais como a cirrose hepática, hepatocarcinoma e necessidade de transplante ${ }^{(6)}$.

Uma das maiores dificuldades a respeito dessa meta está em identificar novos indivíduos infectados com o vírus. Há alguns anos, estudos de rastreios em unidades hospitalares eram questionados, devido à possibilidade de viés. Entretanto, especialmente para o caso da hepatite C, o consenso atual é que testes de rastreios devem ser empregados em locais onde os pacientes estão. Nessa perspectiva, apesar de ainda poucos, vários estudos têm seguido essa estratégia.

Estudos de soroprevalência demonstraram índices bastante elevados de positividade de anticorpos para o $\mathrm{HCV}$ (13\% a $18 \%)$ em algumas populações urbanas de unidade de urgência e emergência ${ }^{(7-8)}$.

A unidade de urgência e emergência atua como uma rede de cuidados de saúde para pacientes de difícil acesso que não procuram serviços de assistência médica fora dessa unidade. Esse local tem demonstrado ser efetivo, tanto para a população de faixas etárias específicas para rastreio, como para o restante da população ${ }^{(9)}$.

Hoje, os pacientes com hepatite $\mathrm{C}$ têm, no Brasil, acesso garantido a um arsenal terapêutico com capacidade de cura em aproximadamente $95 \%$ dos casos. Entretanto, fazer o diagnóstico é um pré-requisito necessário para oferecer esses tratamentos, e a triagem de forma adequada sustenta a cascata de tratamento ${ }^{(10)}$.

Considerando que a hepatite $\mathrm{C}$ representa um grande desafio na saúde pública, o objetivo da presente pesquisa foi identificar estudos que realizaram triagem para hepatite $\mathrm{C}$ e avaliaram a prevalência do vírus em unidades de urgência e emergência.

\section{MÉTODO}

\section{TIPO DE ESTUDO}

Revisão sistemática da literatura. As recomendações PRISMA, a qual preza pela qualidade da revisão sistemática, foram utilizadas para o aperfeiçoamento dos resultados da presente revisão.

\section{CRitérios de SELEÇão}

Foram incluídos estudos que avaliaram a prevalência da hepatite $\mathrm{C}$ em unidades de urgência e emergência. Foram considerados estudos que apresentaram população diversa, inclusive pesquisas que utilizaram amostra segundo a coorte de idade "baby boomers".

Durante a realização da pesquisa, buscou-se, de forma sistemática, identificar artigos, em diferentes bases de dados, que respondessem a questionamentos como: qual a taxa média de publicações relacionadas com o diagnóstico de hepatite $\mathrm{C}$ em unidades de urgência e emergência e qual a prevalência de $\mathrm{HCV}$ nessas unidades de atendimento?

Os resultados a serem expressos nesta pesquisa tratam da utilização de uma nova estratégia baseada no local de rastreio, o que, segundo inúmeros autores, pode, de forma sensível, contribuir de modo mais efetivo com a identificação de pessoas contaminadas pelo HCV.

Por se tratar de uma pesquisa de estudos observacionais, esta revisão se baseou nas recomendações propostas pelo Ministério da Saúde. A pesquisa se baseou no acrônimo $\mathrm{PECO}$, sendo $\mathrm{P}$ a população de interesse (usuários dos serviços de urgência e emergência): $\mathrm{E}$ de exposição (população que foi exposta a métodos de triagem para o $\mathrm{HCV}$ ), $\mathrm{C}$ de controle, esse utilizado para estudo do caso-controle e, por fim, $\mathrm{O}$ de outcome, ou seja, desfecho, que, nesse caso, foi a prevalência do HCV na população descrita ${ }^{(11)}$. O estudo seguiu as seguintes etapas: identificação do tema, das questões de pesquisa e dos objetivos; definição das bases de dados e os critérios para inclusão e exclusão; definição das informações a serem extraídas e categorização; avaliação dos estudos incluídos na revisão por diferentes pesquisadores e interpretação dos resultados; apresentação da revisão e síntese do conhecimento ${ }^{(12)}$.

Não houve restrições quanto ao idioma de publicação. As coletas de dados ocorreram de janeiro a 20 de setembro de 2018.

Para a avaliação qualitativa dos métodos descritos nos estudos observacionais, foram utilizadas as ferramentas do STROBE para estudo de coorte, caso-controle e tranversal ${ }^{(13)}$.

Foram considerados elegíveis estudos de prevalência que avaliaram o método de rastreio segundo o local. Os critérios de inclusão envolveram pessoas de quaisquer idades, desde que atendidas no Pronto Atendimento.

\section{Coleta De DAdos}

As bases de dados utilizadas na busca eletrônica foram: Literatura Latino-Americana e do Caribe em Ciências da Saúde (LILACS), Medical Literature Analysis and Retrieval System (MEDLINE), Scientific Electronic Library Online (SciELO) e Biblioteca Virtual em Saúde (BVS), por meio do site www.bvsalud.org. Ressalta-se que a BVS integra a Biblioteca Cochrane, o Índice Bibliográfico Espanhol de Ciências da Saúde (IBECS).

Para a busca dos artigos, foram utilizados os descritores padronizados no MeSH (PubMed) e em ciências da saúde (DeCS). No MEDLINE, foram utilizados descritores em inglês, e no LILACS e SciELO, português-espanhol (Quadro 1).

A estratégia de busca de alta sensibilidade e os termos e operadores booleanos (OR, $A N D, N O T)$ foram aplicados conforme compatibilidades das bases de dados. 
Quadro 1 - Estratégias de busca estabelecidas para a base de dados PubMed.

1.Hepatitis C* AND Emergency Department* AND Triage*

2. Hepatitis $C^{*} A N D$ Mass Screening* AND Emergency Department*

3. Hepatitis $C^{*}$ AND Screening* AND Emergency Department*

\section{ANÁlISE E TRATAMENTO DOS DADOS}

Dois avaliadores fizeram, separadamente, a leitura dos títulos e resumos de cada referência pré-selecionada, a fim de identificar somente os estudos que preenchiam corretamente os critérios de inclusão. Prosseguiu-se com a leitura dos artigos, separadamente, a fim de assegurar os critérios da revisão sistemática. Em caso de desacordo entre os dois revisores, um terceiro avaliou o manuscrito e, por consenso, foi tomada a decisão para inclusão. A análise dos estudos segundo o nível de evidência se baseou no Oxford Centre Evidence-Based Medicine, sendo IIB estudos de coorte com pobre qualidade de randomização, estudo do tipo controle, estudo sem acompanhamento a longo prazo e também estudo de coorte transversal. O grau de evidência foi obtido a partir da análise individual dos desenhos metodológicos utilizados nas pesquisas.

Os estudos selecionados foram inseridos e organizados em uma planilha do Microsoft Office Excel (2013), com determinados itens de importância para a pesquisa como: título, objetivos, ano de publicação, periódico, desenho do estudo e desfechos.

\section{RESULTADOS}

Com os descritores utilizados, foram identificados 89 artigos na base de dados PubMed. Nenhum artigo foi encontrado nas bases LILACS e SciELO.
Após a avaliação dos títulos e dos resumos, considerando os critérios de inclusão, 19 artigos foram selecionados e 70 excluídos por não contemplarem os critérios pré-estabelecidos. Todos os artigos selecionados foram publicados no idioma inglês.

As datas de publicação se concentraram entre $1992 \mathrm{e}$ 2018, apresentando um intervalo de 26 anos entre a primeira e a última publicação. A média de publicações foi de 2,37/ano.

Em relação ao número e prevalência de publicações por ano, se observa que, em 2018, foram publicados, até o presente momento, três estudos (15\%). Em 2016 foram sete estudos (35\%). Por sua vez, em 2015, dois estudos (10\%) seguidos de três (15\%) em 2014. Para os outros anos (2012, 2002, 1999, 1992), foi publicado apenas 1 estudo $(5,26 \%)$.

Das revistas que mais receberam artigos destacam-se: Annals of Emergency Medicine representando com 5 publicações (26,31\%); a PLoS One e Clinical Infectious Diseases apresentando dois estudos cada $(21,05 \%)$. As demais revistas receberam somente 1 artigo cada.

As informações referentes ao título, autores, país, ano de publicação e base de dados estão disponíveis na Quadro 2.

A respeito do rastreamento segundo a faixa etária "baby boomers", foram identificados apenas quatro estudos procedentes do continente norte-americano. Entende-se por "baby boomers" a geração de pessoas nascidas após a segunda guerra mundial, ou seja, aquelas que nasceram entre 1945 e 1960.

Em relação a publicações conforme a origem, são apresentados: Estados Unidos da América (EUA), com 12 publicações $(63,15 \%)$, Europa, com seis $(31,57 \%)$ e, por fim, a Ásia, com apenas uma publicação $(5,28 \%)$. Em relação à prevalência nos EUA, se observou uma variação entre 1,4\% e 18\%. Por sua vez, na Europa, a prevalência variou de $0 \mathrm{em}$ um serviço de rastreio para migrantes a $5,05 \%$. No estudo asiático, a prevalência foi de $1,8 \%$.

Quadro 2 - Características dos estudos incluídos na revisão sistemática.

\begin{tabular}{|c|c|c|c|c|c|c|}
\hline Título & Autores/Ano & Revista & Delineamento & Resultado/Desfechos & $\begin{array}{l}\text { Base de } \\
\text { dados }\end{array}$ & $\begin{array}{c}\text { Nível de } \\
\text { evidência }\end{array}$ \\
\hline $\begin{array}{l}\text { Hepatitis } B \text { and hepatitis } C \text { in } \\
\text { emergency department patients }\end{array}$ & $\begin{array}{l}\text { Kelen, et al; } \\
1992^{(7)}\end{array}$ & N Engl J Med. & $\begin{array}{c}\text { Estudo transversal } \\
\text { (Prevalência)/Análise } \\
\text { descritiva. }\end{array}$ & Prevalência Anti-HCV 18\% & PubMed & IIB \\
\hline $\begin{array}{l}\text { Prevalence and risk factors } \\
\text { associated with hepatitis } C \text { in } \\
\text { ED patients. }\end{array}$ & $\begin{array}{c}\text { Brillman, et al; } \\
2002^{(8)} \text {. }\end{array}$ & $\begin{array}{l}\text { Am J Emerg } \\
\text { Med. }\end{array}$ & $\begin{array}{c}\text { Estudo transversal } \\
\text { (Prevalência)/Análise } \\
\text { descritiva. }\end{array}$ & Prevalência Anti-HCV 17\%. & PubMed & IIB \\
\hline $\begin{array}{l}\text { A Comparative effectiveness } \\
\text { study of two nontargeted } \\
\text { HIV and Hepatitis C Virus } \\
\text { Screening algorithms in an urban } \\
\text { emergency department }\end{array}$ & $\begin{array}{l}\text { White, et al; } \\
2010^{(14)} \text {. }\end{array}$ & $\begin{array}{l}\text { Ann Emerg } \\
\text { Med. }\end{array}$ & $\begin{array}{l}\text { Estudo transversal } \\
\text { (Prevalência)/ } \\
\text { Estudo de eficácia } \\
\text { comparativa. }\end{array}$ & $\begin{array}{c}\text { Novos diagnósticos de } \\
\text { hepatite C por método } \\
\text { eletrônico } 101(1,44 \%) \text {. } \\
\text { Novos diagnósticos de } \\
\text { hepatite C por método de } \\
\text { triagem tradicional } 9(0,30 \%) \text {. }\end{array}$ & PubMed & IIB \\
\hline $\begin{array}{l}\text { Implementation and preliminary } \\
\text { results of an emergency department } \\
\text { nontargeted, Opt-out Hepatitis C } \\
\text { Virus Screening Program }\end{array}$ & $\begin{array}{l}\text { Schechter- } \\
\text { Perkins, et al; } \\
2018^{(15)} \text {. }\end{array}$ & $\begin{array}{l}\text { Acad Emerg } \\
\text { Med. }\end{array}$ & $\begin{array}{c}\text { Estudo transversal } \\
\text { (Prevalência)/Análise } \\
\text { descritiva. }\end{array}$ & $\begin{array}{c}\text { Prevalência Anti-HCV } \\
13,2 \% \\
\text { Prevalência segundo HCV- } \\
\text { RNA } 7,7 \% \text {. }\end{array}$ & PubMed & IIB \\
\hline $\begin{array}{l}\text { Prevalence of diagnosed and } \\
\text { undiagnosed Hepatitis } C \text { in a } \\
\text { midwestern urban emergency } \\
\text { department }\end{array}$ & $\begin{array}{l}\text { Lyons, et al; } \\
2016^{(16)} \text {. }\end{array}$ & Clin Infect Dis. & $\begin{array}{c}\text { Estudo transversal } \\
\text { (Prevalência)/Análise } \\
\text { descritiva. }\end{array}$ & $\begin{array}{c}\text { Prevalência Anti-HCV 14\% } \\
\text { HCV-RNA } 81 \% .\end{array}$ & PubMed & IIB \\
\hline
\end{tabular}


...continuação

\begin{tabular}{|c|c|c|c|c|c|c|}
\hline Título & Autores/Ano & Revista & Delineamento & Resultado/Desfechos & $\begin{array}{c}\text { Base de } \\
\text { dados }\end{array}$ & $\begin{array}{l}\text { Nível de } \\
\text { evidência }\end{array}$ \\
\hline $\begin{array}{l}\text { Emergency department screening } \\
\text { for Hepatitis C virus: geographic } \\
\text { reach and spatial clustering in } \\
\text { Central Alabama }\end{array}$ & $\begin{array}{l}\text { Donnelly, et } \\
\text { al; } 2016^{(17)} \text {. }\end{array}$ & Clin Infect Dis. & $\begin{array}{l}\text { Estudo de } \\
\text { prevalência/ } \\
\text { retrospectivo. }\end{array}$ & $\begin{array}{c}\text { Prevalência Anti-HCV } \\
11,6 \% .\end{array}$ & PubMed & IIB \\
\hline $\begin{array}{l}\text { Evaluation of the centers for } \\
\text { disease control and prevention } \\
\text { Recommendations for Hepatitis C } \\
\text { virus testing in an urban emergency } \\
\text { department }\end{array}$ & $\begin{array}{l}\text { Hsieh, et al; } \\
2016^{(18)}\end{array}$ & $\begin{array}{l}\text { Am J Emerg } \\
\text { Med. }\end{array}$ & $\begin{array}{l}\text { Estudo transversal } \\
\text { (Prevalência)/Análise } \\
\text { descritiva. }\end{array}$ & $\begin{array}{c}\text { Prevalência Anti-HCV } \\
13,8 \%\end{array}$ & PubMed & IIB \\
\hline $\begin{array}{l}\text { HCV among The Miriam Hospital } \\
\text { and Rhode Island Hospital Adult } \\
\text { ED Patients }\end{array}$ & $\begin{array}{l}\text { Merchant, et } \\
\text { al; } 2014^{(19)} \text {. }\end{array}$ & R I Med J. & $\begin{array}{c}\text { Estudo transversal } \\
\text { (Prevalência)/Análise } \\
\text { descritiva. }\end{array}$ & Prevalência Anti-HCV 4,6\% & PubMed & IIB \\
\hline $\begin{array}{l}\text { Unrecognized chronic hepatitis } \\
\text { C virus infection among baby } \\
\text { boomers in the emergency } \\
\text { department }\end{array}$ & $\begin{array}{l}\text { Galbraith; et } \\
\text { al; } 2015^{(20)} \text {. }\end{array}$ & Hepatology. & $\begin{array}{c}\text { Estudo transversal } \\
\text { (Prevalência)/Análise } \\
\text { descritiva. }\end{array}$ & $\begin{array}{c}\text { Prevalência Anti-HCV } \\
11,1 \% .\end{array}$ & PubMed & IIB \\
\hline $\begin{array}{l}\text { Hepatitis C virus infection in the } \\
1945-1965 \text { birth cohort (baby } \\
\text { boomers) in a large urban ED }\end{array}$ & $\begin{array}{c}\text { Allison, et al; } \\
2016^{(21)}\end{array}$ & $\begin{array}{l}\text { Am J Emerg } \\
\text { Med. }\end{array}$ & $\begin{array}{l}\text { Estudo transversal } \\
\text { (Prevalência)/Análise } \\
\text { descritiva. }\end{array}$ & Prevalência Anti-HCV 7,3\%. & PubMed & IIB \\
\hline $\begin{array}{l}\text { Results of a Hepatitis C virus } \\
\text { screening program of the 1945- } \\
1965 \text { Birth Cohort in a large } \\
\text { emergency department in New } \\
\text { Jersey }\end{array}$ & $\begin{array}{l}\text { Cornett, et al; } \\
2018^{(22)} \text {. }\end{array}$ & $\begin{array}{l}\text { Open Forum } \\
\text { Infect Dis. }\end{array}$ & $\begin{array}{l}\text { Estudo de } \\
\text { prevalência/ } \\
\text { retrospectivo. }\end{array}$ & Prevalência Anti-HCV 6,3\%. & PubMed & IIB \\
\hline $\begin{array}{l}\text { Results of a Rapid Hepatitis C Virus } \\
\text { Screening and Diagnostic Testing } \\
\text { Program in an Urban Emergency } \\
\text { Department }\end{array}$ & $\begin{array}{l}\text { White, et al; } \\
2016^{(23)} \text {. }\end{array}$ & $\begin{array}{l}\text { Ann Emerg } \\
\text { Med. }\end{array}$ & $\begin{array}{l}\text { Estudo de } \\
\text { Prevalência/ } \\
\text { retrospectivo }\end{array}$ & $\begin{array}{c}\text { Prevalência Anti-HCV } \\
10,3 \%\end{array}$ & PubMed & IIB \\
\hline $\begin{array}{l}\text { Incorporating HIV/hepatitis B virus/ } \\
\text { hepatitis C virus combined testing } \\
\text { into routine blood tests in nine } \\
\text { UK Emergency Departments: the } \\
\text { "Going Viral" campaign }\end{array}$ & $\begin{array}{l}\text { Orkin, et al; } \\
2016^{(24)}\end{array}$ & HIV Med. & $\begin{array}{c}\text { Estudo transversal } \\
\text { (Prevalência)/Análise } \\
\text { descritiva. }\end{array}$ & $\begin{array}{c}\text { Prevalência Anti-HCV } \\
2,46 \%\end{array}$ & PubMed & IIB \\
\hline $\begin{array}{l}\text { High prevalence of hepatitis } \\
C(H C V) \text { in the emergency } \\
\text { department (ED) of a London } \\
\text { hospital: should we be screening } \\
\text { for HCV in ED attendees? }\end{array}$ & $\begin{array}{l}\text { Orkin, et al; } \\
2015^{(25)} \text {. }\end{array}$ & $\begin{array}{l}\text { Epidemiol } \\
\text { Infect. }\end{array}$ & $\begin{array}{c}\text { Estudo transversal } \\
\text { (Prevalência)/Análise } \\
\text { descritiva. }\end{array}$ & $\begin{array}{c}\text { Prevalência Anti-HCV 2,6\% } \\
\text { Prevalência total após } \\
\text { análise de HCV-RNA 1,2\%. }\end{array}$ & PubMed & IIB \\
\hline $\begin{array}{l}\text { Screening for latent TB, HIV, and } \\
\text { hepatitis } B / C \text { in new migrants in a } \\
\text { high prevalence area of London, } \\
\text { UK: a cross-sectional study }\end{array}$ & $\begin{array}{l}\text { Hargreaves, et } \\
\text { al; } 2014^{(26)} \text {. }\end{array}$ & $\begin{array}{l}\text { BMC Infect } \\
\text { Dis. }\end{array}$ & $\begin{array}{c}\text { Estudo transversal } \\
\text { (Prevalência)/Análise } \\
\text { descritiva. }\end{array}$ & Prevalência Anti-HCV 0\% & PubMed & IIB \\
\hline $\begin{array}{l}\text { Opt-Out Panel Testing for HIV, } \\
\text { Hepatitis B and Hepatitis C in an } \\
\text { urban emergency department: a } \\
\text { pilot study }\end{array}$ & $\begin{array}{l}\mathrm{O}^{\prime} \text { Connell, et } \\
\text { al; } 2016^{(27)} \text {. }\end{array}$ & PLoS One. & $\begin{array}{c}\text { Estudo transversal } \\
\text { (Prevalência)/Análise } \\
\text { descritiva. }\end{array}$ & $\begin{array}{c}\text { Prevalência Anti-HCV } \\
5,05 \%\end{array}$ & PubMed & IIB \\
\hline $\begin{array}{l}\text { High prevalence of anti-HCV } \\
\text { antibodies in two metropolitan } \\
\text { emergency departments in } \\
\text { Germany: a prospective screening } \\
\text { analysis of } 28,809 \text { patients }\end{array}$ & $\begin{array}{l}\text { Vermehren, et } \\
\text { al; } 2012^{(28)} \text {. }\end{array}$ & PLoS One. & $\begin{array}{c}\text { Estudo transversal } \\
\text { (Prevalência)/Análise } \\
\text { descritiva. }\end{array}$ & $\begin{array}{c}\text { Prevalência Anti-HCV 2,6\% } \\
\text { Prevalência total após } \\
\text { análise de RNA-HCV } \\
1,6 \%\end{array}$ & PubMed & IIB \\
\hline $\begin{array}{l}\text { Hepatitis C virus infection risk } \\
\text { factors in patients admitted in } \\
\text { hospital emergency departments in } \\
\text { Picardy. Value of oriented screening } \\
\text { based on recommendations of the } \\
\text { 'Direction Générale de la Santé' }\end{array}$ & $\begin{array}{c}\text { Capron, et al; } \\
1999^{(29)}\end{array}$ & $\begin{array}{c}\text { Eur J } \\
\text { Gastroenterol }\end{array}$ & $\begin{array}{c}\text { Estudo transversal } \\
\text { (Prevalência)/Análise } \\
\text { descritiva. }\end{array}$ & Prevalência Anti-HCV 2,4\%. & PubMed & IIB \\
\hline $\begin{array}{l}\text { Hepatitis B, Hepatitis C and HIV } \\
\text { seroprevalence in critically ill } \\
\text { emergency medicine department } \\
\text { patients in a tertiary inner city } \\
\text { hospital in Istanbul, Turkey }\end{array}$ & $\begin{array}{l}\text { Ozturk, et al; } \\
2014^{(30)}\end{array}$ & Pak J Med Sci. & $\begin{array}{c}\text { Estudo transversal } \\
\text { (Prevalência)/Análise } \\
\text { descritiva. }\end{array}$ & Prevalência Anti-HCV 5\% & PubMed & IIB \\
\hline
\end{tabular}


ANÁlISE DESCRITIVA SEGUNDO A ORIGEM DO ESTUDO

\section{AMÉRICA dO NORTE}

A primeira publicação identificada sobre o rastreio da hepatite $\mathrm{C}$ em unidades de emergência foi publicada pela New England em 1992, envolvendo 2.523 pacientes. Este estudo observou uma prevalência de $18 \%$. A hepatite $\mathrm{C}$ foi encontrada em 145 dos 175 usuários de drogas intravenosas (83\%). Trinta e seis dos 171 pacientes apresentavam histórico prévio de transfusão sanguínea. Dos 24 homossexuais, cinco foram positivos para o HCV. Entre os homens de cor negra com idade entre 35-44 anos, a soroprevalência de HCV foi de $51 \%{ }^{(7)}$.

Em 2002 pesquisadores da Faculdade de Medicina da Universidade do Novo México desenvolveram, no departamento de emergência, um estudo com o intuito de avaliar a prevalência e fatores de risco associados à hepatite $\mathrm{C}$ nessa unidade. Pessoas com idade acima de 18 anos, que se apresentavam para atendimento, tinham seus sangues retirados como parte da avaliação médica de rotina. Além dessa amostra, um tubo extra para rastreio do $\mathrm{HCV}$ também foi coletado. Uma análise de caso-controle foi usada para identificar os fatores de risco no HCV. Das 223 amostras de sangue, 38 (17\%) foram positivas para anticorpos do $\mathrm{HCV}$. O uso pregresso de drogas injetáveis foi o fator de risco mais significativo associado ao $\mathrm{HCV}$ (OR 858,5, IC 61,8-22,026,5). Os autores concluíram que altas taxas de HCV são identificadas na unidade urgência e emergência de centros urbanos. Esses pesquisadores valorizam a unidade de emergência como local estratégico para a identificação de pessoas contaminadas pelo $\mathrm{HCV}^{(8)}$.

Pesquisadores norte-americanos compararam a eficácia de dois protocolos de rastreio da síndrome da imunodeficiência humana (HIV) e hepatite $\mathrm{C}$ em um departamento de emergência urbana. Pacientes com solicitação laboratorial para pesquisa de HIV automaticamente faziam rastreio para HCV e vice-versa. Fizeram parte deste estudo pacientes entre 18 e 75 anos de idade. $\mathrm{O}$ estudo concluiu que um algoritmo eletrônico, que liga automaticamente a triagem do HIV e do HCV à solicitação laboratorial para pacientes adultos é mais eficaz do que um protocolo clínico orientado por enfermeiros, por exemplo, em relação aos fatores de risco $^{(14)}$.

Outros estudos nessa linha concordam com a oportunidade que a unidade de emergência apresenta para rastreio do HCV. Nesse contexto, foi realizada uma análise descritiva de três meses (novembro de 2016 a janeiro de 2017) de uma triagem de $\mathrm{HCV}$ entre pacientes com, pelo menos, 13 anos de idade, submetidos à flebotomia para fins clínicos. Pesquisa de anti-HCV foi realizada em 3.808 pacientes. A proporção de positividade para o teste HCV foi de 13,2\% (504/3.808). Desses, 97,8\% (493/504) tiveram teste de HCV-RNA realizado. Um total de 292 pacientes apresentaram infecção ativa, com taxa de positividade total de HCV-RNA de 7,7\% (IC95\% = 6,8\% -8,5\%). Daqueles com infecção ativa, 155 (53\%) estavam fora da coorte de nascimentos do Centers for Disease Control and Prevention para aumento do risco de HCV, que consiste em indivíduos nascidos entre 1945 e $1965^{(15)}$.
Em um centro urbano do Meio Oeste, pesquisadores de um hospital de ensino, com 450 leitos hospitalares e com aproximadamente 90.000 atendimentos anuais na unidade de emergência, realizaram um estudo transversal de soroprevalência. Participaram da pesquisa 924 pacientes com idade entre 18 e 64 anos. O ANTI-HCV foi identificado em 128 amostras (14\%). Desses, 44 (34\%) relataram conhecimento sobre a positividade. Cento e três pacientes $(81 \%)$ foram positivos para HCV-RNA. Dois pacientes adicionais eram negativos em relação ao ANTI-HCV, mas com HCV-RNA positivos. A triagem baseada na coorte de idade teria perdido 36/128 (28\%) dos casos com anticorpo detectável e 26/105 (25\%) daqueles com infecção viral ativa. Os autores concluem que o rastreio para hepatite $\mathrm{C}$ no departamento de emergência é uma estratégia a ser adotada e que se tiverem se pautado na triagem baseada na coorte de nascimento, teriam perdido um número considerável de exames positivos ${ }^{(16)}$.

No Alabama, pesquisadores examinaram os resultados da triagem do HCV no Pronto Atendimento. Esta pesquisa foi realizada na Universidade do Alabama, em Birmingham. Essa instituição tinha como rotina a realização do rastreio da hepatite $\mathrm{C}$ na população "baby boomers"e também nas de alto risco (usuários de drogas intravenosas). Foram excluídos do estudo pacientes já diagnosticados ${ }^{(17)}$. Foram realizados 8.742 testes. Da amostra total, 6.888 nasceram entre 1945-1965 (79\%). A prevalência geral foi $11,6 \%$. Os autores defendem que a triagem em Pronto Atendimento é adequada e que tem a capacidade de impactar a epidemia do HCV através do amplo raio geográfico ${ }^{(17)}$.

Continuando a estratégia de rastreio em unidades específicas, outros pesquisadores realizaram estudo de prevalência com amostras de 4.713 usuários de um serviço de emergência em um centro urbano, identificando uma prevalência de 13,8\% (652). Desses, 204 (31,3\%) não disponibilizavam nenhum registro quanto à infecção por HCV. Dos pacientes com infecção não documentada, 99 (48,5\%) foram diagnosticados com base na coorte do nascimento e $54(26,5 \%)$ por testes baseados nos fatores de risco. Os autores concluíram que se a triagem fosse realizada seguindo as orientações por faixa etária, $51(25 \%)$ dos pacientes com HCV não seriam testados ${ }^{(18)}$.

Em 2014, pesquisadores estimaram a prevalência de $\mathrm{HCV}$ em pacientes adultos, bem como entre pacientes com qualquer relato de uso de drogas injetáveis em um hospital de uma ilha nos EUA. A taxa de positividade do teste de $\mathrm{HCV}$ entre 8.500 pacientes adultos foi de $4,6 \%$ e a soroprevalência de anti-HCV entre 621 pacientes adultos que usaram drogas, foi de $1,6 \%$. Dentre os dez pacientes com diagnóstico de $\mathrm{HCV}$ positivo não diagnosticado anteriormente, oito nasceram após 1965 e seis nunca receberam drogas injetáveis ${ }^{(19)}$.

Outros estudos avaliaram a prevalência do $\mathrm{HCV}$ em unidades de urgência e emergência com base na faixa etária. Em 2015, realizaram uma das primeiras pesquisas para rastreio do HCV, seguindo as recomendações do CDC quanto aos "baby boomers". Participaram da pesquisa 1.529 indivíduos, desses, 170 (11,1\%) foram reagentes para o HCV. O HCV-RNA foi realizado em 150 pacientes $(88,2 \%)$, dos quais, $102(68,0 \%)$ foram confirmados. A soroprevalência foi 
maior em homens do que em mulheres $(14,7 \%$ versus $7,4 \%$, $\mathrm{P}<0,001)$ e em afrodescendentes quando comparados aos brancos $(13,3 \% \text { versus } 8,8 \%, \mathrm{P}=0,010)^{(20)}$.

Um outro estudo que também seguiu a recomendação de rastreio baseado na idade, abordou um total de 915 pacientes para a triagem do HCV. Desses, 427 participaram de uma entrevista estruturada e 383 concordaram em realizar um teste rápido. A prevalência da $\mathrm{HCV}$ foi de 7,3\%. Foram observados equívocos em relação ao conhecimento da transmissão da doença entre a população pesquisada ${ }^{(21)}$.

Ainda nesse contexto de "baby boomers", foi desenvolvido numa unidade de emergência fora do centro urbano de Nova Jersey, um estudo de coorte retrospectivo de pacientes nascidos entre 1945-1965 testados para HCV. O objetivo foi avaliar a prevalência da infecção pelo HCV e analisar as características associadas aos resultados positivos. De um total de 3.046 pacientes, 192 foram positivos para anticorpos (6,3\%). Dos 167 com resultados do teste de carga viral, $43 \%$ eram virêmicos ${ }^{(22)}$.

$\mathrm{Na}$ Califórnia, pesquisadores realizaram um estudo retrospectivo no departamento de emergência de um hospital do centro urbano, o qual realiza em torno de 90.000 atendimentos por ano. $\mathrm{O}$ objetivo da pesquisa foi avaliar um programa de teste de hepatite $\mathrm{C}$ a partir da coorte de idade e também do histórico de risco. Participaram do estudo pacientes nascidos entre 1945 e 1965. Dos 26.639 adultos com idade igual ou superior a 18 anos que se apresentaram ao departamento de emergência nos últimos 6 meses, 2.581 $(9,7 \%)$ completaram os critérios para rastreio do $\mathrm{HCV}$, dos quais, 267 apresentaram anticorpos positivos, resultando numa prevalência de $10,3 \%$. Foram identificados como fatores de risco, o uso de drogas injetáveis, pessoas sem moradia, idade e gênero masculino. A pesquisa defendeu que esforços para o desenvolvimento de políticas para triagem do $\mathrm{HCV}$ em unidades de emergência devem ser permanentes ${ }^{(23)}$.

\section{EUROPA}

Um estudo realizado no Reino Unido descreveu os resultados de um programa de rastreio para HIV e também para hepatites B e C conceituado de "Going Viral". Essa campanha ocorreu no mês de outubro de 2014 em nove departamentos de emergência, os quais costumavam apresentar uma alta prevalência de HIV. Os testes de rastreio foram oferecidos para os pacientes que receberam hemoderivados como parte do tratamento de rotina. Um total de 7.807 pacientes tiveram sangue coletado em sua visita a unidade de emergência. Em relação ao HCV, foram identificados 39 casos, sendo 15 recém diagnosticados. Houve 17 infecções por HIV (6 recém diagnosticadas) e 15 infecções por HBV (11 recém-diagnosticados). Os autores concluíram que através de uma campanha de detecção, foi possível a identificação de novos casos de doenças virais de importância pública, encorajando que a realização de triagens em unidades de emergência parece ser uma boa estratégia para o rastreio de diferentes doenças virais simultaneamente ${ }^{(24)}$.

Ainda no Reino Unido, outros pesquisadores, a partir de 997 amostras colhidas ao longo de 12 dias para a detecção de anticorpo de $\mathrm{HCV}$ e também para RNA-HCV, identificaram uma prevalência anti-HCV de 2,6\% (26/997) com $1,2 \%$ para o $\mathrm{RNA}^{(25)}$.

Em 2016, ainda no cenário britânico, pesquisadores realizaram triagem para tuberculose, vírus $\mathrm{B}$ e $\mathrm{C}$ da hepatite em dois diferentes departamentos de emergência. Esse movimento foi articulado mediante o aumento significativo de imigrantes. A intervenção aumentou o diagnóstico de tuberculose latente, entretanto nenhum caso de $\mathrm{HIV}$ ou hepatite $\mathrm{B} / \mathrm{C}$ foi encontrado ${ }^{(26)}$.

$\mathrm{Na}$ Irlanda, pesquisadores avaliaram a viabilidade e aceitabilidade de um programa universal de exclusão de HIV, hepatite $\mathrm{B}$ e $\mathrm{C}$ em um departamento de emergência de um grande hospital, em pacientes com idade igual ou superior a 18 anos. Os autores descreveram ainda a incidência e a prevalência das morbidades citadas. Os pacientes foram submetidos a amostragem de sangue durante a rotina de tratamento clínico. Com mais de 45 semanas de testes, 8.839 amostras individuais foram disponibilizadas para a análise. A incidência da $\mathrm{HCV}$ foi de 6,5 para cada 1.000 habitantes com uma prevalência de 5,05\%. A pesquisa concluiu que o rastreio para doenças virais é uma estratégia viável e aceitável nas unidades de emergência ${ }^{(27)}$.

$\mathrm{Na}$ Alemanha, foi avaliada a soroprevalência de $\mathrm{HCV}$ em pacientes que visitaram dois serviços de emergência terciária em Berlim e Frankfurt, respectivamente. Um total de 28.809 pacientes foram rastreados quanto a identificação de anticorpos anti-HCV. Os soros anti-HCV positivos foram subsequentemente testados para $\mathrm{HCV}$-RNA. A soroprevalência global do $\mathrm{HCV}$ foi de 2,6\%. O HCV-RNA foi detectável em $68 \%$ dos casos positivos anti-HCV. A prevalência de infecção crônica pelo $\mathrm{HCV}$ na população total do estudo foi de 1,6\% (IC 95\%: 1,5-1,8). O fator de risco mais presente foi o uso de drogas injetáveis $(31,2 \%)$. A população mais jovem da amostra foi responsável por apresentar o principal fator de risco para HCV $(\mathrm{p}<0,001)$ e a relação homem-mulher foi de $72 \%$ (121 versus 46 pacientes, $\mathrm{p}<0,001)$. Finalmente, $18,8 \%$ dos pacientes positivos com HCV-RNA não foram diagnosticados previamente ${ }^{(28)}$.

$\mathrm{Na}$ França, outros pesquisadores estimaram a frequência de fatores de risco em pacientes admitidos na unidade de emergência de sete hospitais de Picardia. Entre os 1.648 pacientes, $68,7 \%$ apresentaram pelo menos um fator de risco. A triagem foi aceita por 723 pacientes, desses, 58,7\% relataram pelo menos um fator de risco, sendo $70 \%$ com histórico de hemotransfusão pregressa ou uso de drogas injetáveis. $\mathrm{O}$ rastreio em 451 usuários evidenciou que 2,4\% tinham anti-HCV positivo. A prevalência do anti-HCV foi de 1,5\% em pacientes sem história de transfusão ou uso de drogas e 7,9\% naqueles com pelo menos um desses dois fatores de risco. $\mathrm{O}$ estudo concluiu que um rastreio baseado em fatores de risco como transfusão sanguínea e uso de drogas injetáveis, parece ter uma melhor eficiência ${ }^{(29)}$.

\section{Ásı}

Um estudo avaliou a prevalência da hepatite $\mathrm{B}$, hepatite C e HIV em pacientes com diagnóstico clínico crítico, classificados em cor vermelha no departamento de emergência de um hospital terciário em Istambul na Turquia. Participaram 
da pesquisa 1.000 pacientes. Desses, 50 foram positivos para $\mathrm{HBV}(5 \%), 18$ para $\mathrm{HCV}(1,8 \%)$ e 2 com coinfecção HBVHCV. O HIV não foi registrado. Quarenta e um desses pacientes foram vítimas de trauma, 226 estavam inconscientes ou não cooperativos. Noventa e dois pacientes $(9,2 \%)$ tinham histórico de transfusão sanguínea e entre esses, 11 tinham o diagnóstico de $\mathrm{HBV}$ e 3 de HCV. O estudo concluiu que a soroprevalência no grupo de estudo foi muito baixa, relacionando o resultado com a literatura turca ${ }^{(30)}$.

\section{DISCUSSÃO}

A partir da análise de todos os estudos, fica evidente que a realização do rastreio para a hepatite $\mathrm{C}$ é uma tendência, a qual deve transcender as regiões geográficas aqui mencionada.

É notório que os Estados Unidos contam com o maior número de publicações e isso evidencia a preocupação deste país com o enfrentamento da hepatite $\mathrm{C}$, especialmente com as potenciais complicações.

No Brasil não foi identificado até o presente momento nenhuma publicação que cumprisse o objetivo desta revisão.

Diversas estratégias vêm sendo adotadas com o intuito de se identificar pessoas contaminadas pelo $\mathrm{HCV}$, muitas delas elaboradas e incentivadas pelo Center Disease Control, como exemplo a estratégia dos “baby boomers", que recomenda que pessoas que nasceram entre 1945 e 1965 realizem o teste diagnóstico, por entenderem que essa população apresenta um elevado risco de apresentar o vírus da hepatite ${ }^{(18)}$.

Alguns estudos adotaram essa medida, somada à estratégia relacionada ao local do teste, nesse caso o Pronto Atendimento. Os estudos aqui apresentados relataram uma prevalência que variou de 6,5 a $11 \%{ }^{(18-21)}$.

Apesar de até o momento não terem sido identificadas no Brasil, publicações relacionadas à triagem do $\mathrm{HCV}$ em unidades de urgência, uma pesquisa desenvolvida em uma Unidade Básica de Saúde do estado do Paraná, identificou uma prevalência também abaixo do que se esperava $(0,30 \%)$, sendo mais comum na população com 40 anos de idade ou mais ${ }^{(31)}$.

Há uma grande discussão de qual método de rastreio é mais adequado. Trata-se de uma questão intrigante, difícil de ser respondida em virtude da escassez de publicações. Entretanto, diferentes métodos são necessários, uma vez que nos EUA estima-se que $43 \%$ a $72 \%$ das infecções por HCV não são diagnosticadas ${ }^{(32-34)}$. Além disso, do total da população de indivíduos contaminados pelo $\mathrm{HCV}$ de um país de alta renda, apenas 34\% fazem parte de um grupo de alto risco, ou seja, são mais acessíveis a rastreios ${ }^{(35)}$.

Uma revisão sistemática a respeito dos diferentes métodos empregados para o reconhecimento de pessoas infectadas identificou, a partir de 7.052 estudos, 67 programas de triagem. Desses, 41 eram integrados a serviços de saúde. Os autores concluíram que a maior prevalência de $\mathrm{HCV}$ foi encontrada em programas de países com prevalência intermediária para $\mathrm{HCV}$, em clínicas psiquiátricas e em programas que usaram uma seleção de pré-rastreamento com base nos fatores de risco para $\mathrm{HCV}^{(36)}$. Este estudo trouxe em seu escopo poucos trabalhos que utilizaram a unidade de emergência como local estratégico, parte disso se justifica por ter sido publicado em 2014 e a maior parte dos que utilizaram as unidades de emergência foram publicados em $2016^{(36)}$.

Diante os resultados aqui expressos, sugere-se que o método combinado, unindo o rastreio baseado em fatores de risco, faixa etária e local de realização, parece ser o mais adequado à realidade epidemiológica atual. Essa afirmação é a soma dos estudos que utilizaram o Pronto Atendimento com a inclusão de diferentes faixa-etárias e fatores de risco e também para aquelas que utilizaram a unidade de emergência com a política dos "baby boomers”. Em uma pesquisa que utilizou como estratégia a unidade de emergência mais a política da faixa etária e mais os riscos conhecidos, observou que $79 \%$ dos resultados positivos estavam ligados aos nascidos entre 1945 e $1965^{(17)}$. Uma outra justificativa para a realização da triagem em unidade de emergência é a possível capacidade de alcance geográfico que essa unidade apresenta ${ }^{(14)}$. Por outro lado, restringir a triagem para hepatite $\mathrm{C}$ para a faixa-etária, pode ser responsável por perder em torno de $25 \%$ a $45 \%$ dos pacientes infectados ${ }^{(16-17)}$.

Para detalhar qual método seria mais apropriado, deve-se discutir alguns pontos importantes. Diversos estudos estão com seus protocolos bem delimitados, alguns utilizam faixa-etária mais fatores de risco conhecidos, outros apenas a faixa-etária. Além, é claro, da metodologia do protocolo em si, há elementos nesse processo que são fundamentais para o sucesso do método: a maneira como se oferecem os exames. Autores descrevem que muitos enfermeiros da triagem ofereceram o teste do $\mathrm{HCV}$ a mais de 2.000 pacientes fora da coorte de nascimento e cujo status de uso de drogas injetáveis era desconhecido. Também enfatizam que muitos médicos solicitam os testes para HCV baseados em seus critérios de formação. Esse tipo de julgamento está à mercê de cometer equívocos e falhar na identificação de novos $\operatorname{casos}^{(23)}$.

Para cumprir os critérios de rastreio, minimizando viés de oferta, pesquisadores defendem maior efetividade na detecção do $\mathrm{HCV}$ a partir de um meio eletrônico ${ }^{(14)}$. $\mathrm{O}$ algoritmo automatizado de rastreio por laboratório analisou mais de $33 \%$ dos doentes do departamento de emergência elegíveis para o HCV em comparação com menos de $20 \%$ dos pacientes com o algoritmo de rastreamento do vírus hepatite $\mathrm{C}^{(14,30)}$.

Com esta revisão, buscou-se apresentar o panorama sobre publicações relacionadas aos métodos de triagem empregados na identificação de novos pacientes portadores de hepatite $\mathrm{C}$. Até o presente momento, não foram encontradas publicações nacionais relacionadas ao tema para descrever e sustentar, ou não, as melhores práticas no país. Novas pesquisas devem ser realizadas, dentro da perspectiva de se implementar práticas sensíveis às necessidades de saúde da população.

\section{CONCLUSÃO}

O rastreio realizado em unidades de urgência e emergência se mostrou eficiente na identificação de novos casos, especialmente quando associado ao fator idade, que demonstra ser superior à estratégia baseada apenas em fatores de risco. Assim, para maior efetividade, recomenda-se a associação de três estratégias: rastreio em unidades de urgência e emergência, com base na faixa etária e identificação de fatores de risco. 
RESUMO

Objetivo: Identificar estudos que realizaram rastreio para hepatite $\mathrm{C}$ e que também avaliaram a prevalência do vírus em usuários de unidades de urgência e emergência. Método: Revisão sistemática realizada nas bases de dados LILACS, MEDLINE via PubMed, SciELO e Biblioteca Virtual em Saúde. Resultados: Após criteriosa busca e análise, fizeram parte dos resultados 19 publicações, essas em conformidades com os critérios pré-definidos. As datas de publicação se concentraram entre 1992 e 2018 . A principal revista a publicar tais artigos foi a Annals of Emergency Medicine (26,31\%). Em relação à origem das publicações, destacam-se: Estados Unidos (63,15\%), Europa (31,57\%) e Ásia (5,28\%). Em relação à prevalência, nos Estados Unidos, se observou uma variação entre 1,4\% e 18\%. Por sua vez, na Europa, a prevalência variou de 0 a $5 \%$. No estudo asiático, a prevalência foi de 1,8\%. Conclusão: O rastreio realizado em unidades de urgência e emergência se mostrou eficiente na identificação de novos casos, especialmente quando associado ao fator idade, que demonstra ser superior à estratégia baseada apenas em fatores de risco. Assim, para maior efetividade, recomenda-se a associação das três estratégias: local do rastreio, faixa etária e fatores de risco.

\section{DESCRITORES}

Hepatite C; Programas de Rastreamento; Prevalência; Assistência Ambulatorial; Revisão Sistemática.

\section{RESUMEN}

Objetivo: Identificar los estudios que realizaron pruebas de detección de hepatitis $\mathrm{C}$ y que también evaluaron la prevalencia del vírus en usuarios de unidades de urgencia y emergencia. Método: revisión sistemática realizada en bases de datos LILACS, MEDLINE a través de PubMed, SciELO y la Biblioteca Virtual en Salud. Resultados: Después de una cuidadosa búsqueda y análisis, 19 publicaciones fueron parte de los resultados, estos cumplieron con los criterios predefinidos. Las fechas de publicación se concentraron entre 1992 y 2018. La revista principal para publicar dichos artículos fue el Annals of Emergency Medicine (26,31\%). En cuanto al origen de las publicaciones, destacan las siguientes: Estados Unidos (63,15\%), Europa (31,57\%) y Asia $(5,28 \%)$. Con respecto a la prevalencia, en los Estados Unidos, se observó una variación entre 1.4\% y 18\%. En Europa, a su vez, la prevalencia varió de 0 a $5 \%$. En el estudio asiático, la prevalencia fue de 1,8\%. Conclusión: La evaluación realizada en las unidades de urgencias y emergencias demostró ser eficiente en la identificación de nuevos casos, especialmente cuando se asocia con el factor de edad, que demuestra ser superior a la estrategia basada solo en factores de riesgo. Por lo tanto, para una mayor efectividad, se recomienda la asociación de las tres estrategias: ubicación del examen, grupo de edad y factores de riesgo.

\section{DESCRIPTORES}

Hepatitis C; Tamizaje Massivo; Prevalencia; Atención Ambulatoria; Revisión Sistemática.

\section{REFERÊNCIAS}

1. Gower E, Chris E, Blach S, Razavi-Shearer K, Razavi H. Global epidemiology and genotype distribution of the hepatitis $C$ virus infection. J Hepatol. 2014;61(1 Supll):S45-57. DOI: http://10.1016/j.jhep.2014.07.027

2. Popping S, El-Sayed M, Feld J, Hatzakis A, Hellard M, Les O. Report from the International Viral Hepatitis Elimination Meeting (IVHEM), 17-18 November 2017, Amsterdam, the Netherlands: gaps and challenges in the WHO 2030 hepatitis C elimination framework. J Virus Erad. 2018;4(3):193-5.

3. Universidade de Pernambuco. Núcleo de Pós-Graduação. Estudo de prevalência de base populacional das infecções pelos vírus das hepatites A, B e C nas capitais do Brasil. Relatório de Pesquisa [Internet]. Pernambuco; 2012 [citado 2018 ago. 15]. Disponível em: http:// www.aids.gov.br/publicacao/2010/estudo_de_prevalencia_de_base_populacional_das_iinfeccoe_pelos_virus_das_hepatites_b

4. Razavi-Shearer D, Gamkrelidze I, Nguyen MH, Chen D-S, Van Damme P, Abbas Z, et al. Global prevalence, treatment, and prevention of hepatitis B virus infection in 2016: a modelling study. Lancet Gastroenterol Hepatol. 2018;3(6):383-403. DOI: http//10.1016/S2468-1253(18)30056-6

5. Brasil. Ministério da Saúde. Plano para erradicação da hepatite C [Internet]. Brasília; 2018 [citado 2018 ago. 15]. Disponível em: http:// www.aids.gov.br/pt-br/pub/2019/plano-para-eliminacao-da-hepatite-c-no-brasil

6. World Health Organization. Global Health Sector Strategies Viral Hepatitis 2016-2021 [Internet]. Geneva; 2016 [cited 2018 Sep 25]. Available from: http://www.who.int/hepatitis/strategy2016-2021/Draft_global_health_sector_strategy_viral_hepatitis_13nov.pdf

7. Kelen GD, Green GB, Purcell RH, Chan DW, Qaqish BF, Sivertson KT, et al. Hepatitis B and hepatitis C in emergency department patients. N Engl J Med [Internet]. 1992 [cited 2018 Nov 10];326(21):1399-404. Available from: https://www.nejm.org/doi/full/10.1056/ NEJM199205213262105

8. Brillman JC, Crandall CS, Florence CS, Jacobs JL. Prevalence and risk factors associated with hepatitis C in ED patients. Am J Emerg Med. 2002;20(5):476-80.

9. Hock MOE, Ornato JP, Cosby C, Franck T. Should the emergency department be society's health safety net? J Public Health Policy. 2005;26(3):269- 81. DOI: 10.1057/palgrave.jphp.3200028

10. Jesudian $A B$, Gambarin-Gelwan $M$, Jacobson IM. Advances in the treatment of hepatitis $C$ virus infection. Gastroenterol Hepatol ( $N$ Y). 2012;8(2):91-101.

11. Brasil. Ministério da Saúde. Diretrizes metodológicas de revisão sistemática e metanálise de estudos observacionais comparativos sobre fatores de risco e prognóstico [Internet]. Brasilia; 2014 [citado 2018 set. 15]. Disponível em: http://bvsms.saude.gov.br/bvs/publicacoes/ diretrizes_metodologicas_fatores_risco_prognostico.pdf

12. Whittemore R, Knalf K. The integrative review: update methodology. J Adv Nurs. 2005;52(5):546-53. DOI: 10.1111/j.13652648.2005.03621.x

13. STROBE Statement. Strengthening the reporting of observational studies in epidemiology: checklist of items that should be included in reports of observational studies [Internet]. 2007 [cited 2018 Aug 15]. Available from: https://www.strobe-statement.org/fileadmin/Strobe/ uploads/checklists/STROBE_checklist_v4_combined.doc

14. White DAE, Todorovic T, Petti ML, Ellis KH, Anderson ES. A Comparative effectiveness study of two nontargeted HIV and Hepatitis $\mathrm{C}$ virus screening algorithms in an urban emergency department. Ann Emerg Med. 2018;72(4):438-48. DOl:http// doi: 10.1016/j. annemergmed.2018.05.005 
15. Schechter-Perkins EM, Miller NS, Hall J, Hartman JJ, Dorfman DH, Andry C, et al. Implementation and preliminary results of an emergency department nontargeted, Opt-out Hepatitis C Virus Screening Program. Acad Emerg Med. 2018;25(11):1216-26. DOI: http://dx.doi. org/10.1111/acem.13484

16. Lyons MS, Kunnathur VA, Rouster SD, Hart KW, Sperling MI, Fichtenbaum CJ, Sherman KE. Prevalence of diagnosed and undiagnosed Hepatitis C in a midwestern urban emergency department. Clin Infect Dis. 2016;62(9):1066-71. DOI: http//10.1093/cid/ciw073

17. Donnelly JP, Franco RA, Wang HE, Galbraith JW. Emergency department screening for Hepatitis C Virus: geographic reach and spatial clustering in Central Alabama. Clin Infect Dis. 2016;62(5):613-6. DOI: http// 10.1093/cid/civ984

18. Hsieh $\mathrm{YH}$, Rothman RE, Laeyendecker OB, Kelen GD, Avornu A, Patel EU, et al. Evaluation of the centers for disease control and prevention ecommendations for Hepatitis CVirus testing urban emergency department. Clin Infect Dis. 2016;62(9):1059-65. DOI: http// 10.1093/cid/ciw074

19. Merchant RC, Baird JR, Liu T, Taylor LE. HCV among the Miriam Hospital and Rhode Island Hospital adult ED patients. R I Med J. 2014;97(7):35-9.

20. Galbraith JW, Franco RA, Donnelly JP, Rodgers JB, Morgan JM, Viles AF, et al. Unrecognized chronic hepatitis C virus infection among baby boomers in the emergency department. Hepatology. 2015;61(3):776-82. DOI: http// 10.1002/hep.27410

21. Allison WE, Chiang W, Rubin A, O'Donnell L, Saldivar MA, Maurantonio M, et al. Hepatitis C virus infection in the 1945-1965 birth cohort (baby boomers) in a large urban ED. Am J Emerg Med. 2016;34(4):697-701. DOI: http//10.1016/j.ajem.2015.12.072

22. Cornett JK, Bodiwala V, Razuk V, Shukla D, Narayanan N. Results of a Hepatitis C Virus Screening Program of the 1945-1965 Birth Cohort in a large emergency department in New Jersey. Open Forum Infect Dis. 2018;5(4): ofy065. DOI: 10.1093/ofid/ofy065

23. White DA, Anderson ES, Pfeil SK, Trivedi TK, Alter HJ. Results of a rapid hepatitis $\mathrm{C}$ virus screening and diagnostic testing program in an urban Emergency Department. Ann Emerg Med. 2016;67(1):119-28. DOI: 10.1016/j.annemergmed.2015

24. Orkin C, Leach E, Flanagan S, Wallis E, Ruf M, Foster GR, et al. High prevalence of hepatitis C (HCV) in the emergency department (ED) of a London hospital: should we be screening for HCV in ED attendees? Epidemiol Infect. 2015;143(13):2837-40. DOI: 10.1017/ S0950268815000199

25. Orkin C, Jeffrey-Smith A, Foster GR. Retrospective hepatitis C seroprevalence screening in the antenatal setting-should we be screening antenatal women? BMJ Open. 2016;6(5):e010661. DOl: http://dx.doi.org/10.1136/bmjopen-2015-010661

26. Hargreaves S, Seedat F, Car J, Escombe R, Hasan S, Eliahoo, J, et al. Screening for latent TB, HIV, and hepatitis B/C in new migrants in a high prevalence area of London, UK: a cross-sectional study. BMC Infect Dis. 2014;14:657. DOI: http// 10.1186/s12879-014-0657-2

27. O'Connell S, Lillis D, Cotter A, O'Dea S, Tuite H, Fleming C, et al. Opt-out panel testing for HIV, hepatitis B and hepatitis C in an urban Emergency Department: a pilot study. PLoS One. 2016;11(3):e0150546. DOI: 10.1371/journal.pone.0150546

28. Vermehren J, Schlosser B, Domke D, Elanjimattom S, Müller C, Hintereder G, et al. High prevalence of anti-HCV antibodies in two metropolitan emergency departments in Germany: a prospective screening analysis of 28,809 patients. PLoS One. 2012;7(7):e41206. DOI: 10.1371/journal.pone.0041206

29. Capron D, Bensousan T, Darchis JP, Barbare JC, Butel J, Bental A, et al. Hepatitis C virus infection risk factors in patients admitted in hospital emergency departments in Picardy. Value of oriented screening based on recommendations of the 'Direction Générale de la Santé'. Eur J Gastroenterol Hepatol. 1999;11(6):643-8. DOI: 10.1097/00042737-199906000-00010

30. Ozturk TC, Guneysel O, Tali A, Yildirim SE, Onur OE, Yaylaci S. Hepatitis B, Hepatitis C and HIV seroprevalence in critically ill emergency medicine department patients in a tertiary inner city hospital in Istanbul, Turkey. Pak J Med Sci. 2014;30(4):703-7. DOI: 10.12669/ pjms.304.4975

31. Rodrigues Neto J, Cubas MR, Kusma SZ, Olandoski M. Prevalência da hepatite viral C em adultos usuários de serviço público de saúde do município de São José dos Pinhais - Paraná. Rev Bras Epidemiol. 2012;15(30):627-38. DOI: http://dx.doi.org/10.1590/S1415790X2012000300016

32. Smith BD, Morgan RL, Beckett GA, Falck-Ytter Y, Holtzman D, Teo CG, et al. Recommendations for the identification of chronic hepatitis C virus infection among persons born during 1945-1965. MMWR Recomm Rep. 2012;61(RR-4):1-32.

33. Spradling PR, Rupp L, Moorman AC, Lu M, Teshale EH, Gordon SC, et al. Hepatitis B and C virus infection among 1.2 million persons with access to care: factors associated with testing and infection prevalence. Clin Infect Dis. 2012;55(8):1047-55. DOI: http//10.1093/cid/cis616

34. Volk ML, Tocco R, Saini S, Lok ASF. Public health impact of antiviral therapy for hepatitis C in the United States. Hepatology. 2009;509(6):1750-5. DOI: http:// 10.1002/hep.23220

35. Michitaka K, Horiike N, Ohta Y. An epidemiological study of hepatitis C virus infection in a local district in Japan. Rinsho Byori. 1991;39(6):586-91.

36. Zuure FR, Urbanus AT, Langendam MW, Helsper CW, Van Den Berg CHSB, Davidovich U, et al. Outcomes of hepatitis C screening programs targeted at risk groups hidden in the general population: a systematic review. BMC Public Health. 2014;14:66. DOI: http://10.1186/1471-2458-14-66 